Pacific

Journal of

Mathematics

THE MEROMORPHIC CONTINUATION OF THE RESOLVENT OF THE LAPLACIAN ON LINE BUNDLES OVER $\mathbb{C} H(n)$

Cynthia E. Will 


\title{
THE MEROMORPHIC CONTINUATION OF THE RESOLVENT OF THE LAPLACIAN ON LINE BUNDLES OVER $\mathbb{C} H(n)$
}

\author{
Cynthia E. Will
}

Let $G=S U(n, 1), K=S(U(n) \times U(1))$, and for $l \in \mathbb{Z}$, let $\left\{\tau_{l}\right\}_{l \in \mathbb{Z}}$ be a one-dimensional $K$-type and let $E_{l}$ the line bundle over $G / K$ associated to $\tau_{l}$. In this work we prove that the resolvent of the Laplacian, acting on $C_{c}^{\infty}$-sections of $E_{l}$ is given by convolution with a kernel which has a meromorphic continuation to $\mathbb{C}$. We prove that this extension has only simple poles and we identify the images of the corresponding residues with $(\mathfrak{g}, K)$-submodules of the principal series representations. We show that for certain values of the parameters these modules are holomorphic (or antiholomorphic) discrete series.

\section{Introduction.}

In [9] the meromorphic continuation of the resolvent kernel of the Laplacian acting on functions was studied in the case of the so called Damek-Ricci spaces. These include, in particular, symmetric spaces of strictly negative curvature. This meromorphic continuation has simple poles and the residues are finite rank operators whose images can be explicitly described and their dimensions determined. In the present paper, we shall prove similar results in the case of the action of the Laplacian on line bundles over $\mathbf{C} H^{n}$. We use work by Shimeno on the theory of spherical functions in this context. We prove that in a certain open half-plane of $\mathbb{C}$, the resolvent is given by convolution with an explicit kernel and this has a meromorphic continuation to $\mathbb{C}$. We prove that this continuation has simple poles located at parameters of reducibility of certain principal series representations of $G$. The corresponding residues are convolution operators and their images are isomorphic to $(\mathfrak{g}, K)$-submodules of the principal series representations. For some values of the parameters, these modules are finite dimensional and for others they are holomorphic, antiholomorphic or limits of discrete series representations, hence infinite dimensional. This is in contrast with the case of the trivial $K$-type, studied in [9].

An outline of the paper is as follows: In Sections 2 and 3 we introduce notation and describe some results due mainly to Shimeno, to be used in 
the rest of the paper. In Section 4 we study the meromorphic continuation of the resolvent kernel, and we describe the images of the residues as $(\mathfrak{g}, K)$-submodules of the principal series. We show in particular that any holomorphic or anti-holomorphic representation occurs as image of a residue, as well as any limit of discrete series and any finite dimensional representation whose contain a one dimensional $K$-type. Moreover, in the last case, we also prove that this module is the kernel of the standard intertwining operator of the principal series representation.

We finally discuss in Section 5, in detail, the case when $G=S U(1,1)$.

This paper is part of my thesis work. I am very grateful to my advisor, R. Miatello, for his guidance and constant support. I also wish to thank J. Vargas for helpful conversations.

\section{Preliminaries.}

2.1. Basic notation. We begin by introducing notation that will be used throughout this paper. As is customary, we will denote a Lie group by an upper case letter and its Lie algebra by the corresponding lower case gothic letter.

If $G=\mathrm{S} U(n, 1)$, then the Lie algebra of $G$ is given by $\mathfrak{g}=\{X \in \mathfrak{s l}(n+$ $\left.1, \mathbb{C}): X J+J \bar{X}^{t}=0\right\}$, where $J=\left[\begin{array}{ccc}0 & 0 & 1 \\ 0 & \text { Id } & 0 \\ 1 & 0 & 0\end{array}\right]$.

Let $\mathfrak{g}=\mathfrak{k}+\mathfrak{p}$ be the Cartan decomposition associated to the Cartan involution $\theta(X)=\bar{X}^{t}$. Thus

$$
\begin{aligned}
& \mathfrak{k}=\left\{\left[\begin{array}{cc}
A & 0 \\
0 & y
\end{array}\right]: A \in \mathfrak{u}(n), \operatorname{tr}(A)+y=0\right\} \quad \text { and } \\
& \mathfrak{p}=\left\{\left[\begin{array}{cc}
0 & b \\
\bar{b}^{t} & 0
\end{array}\right]: b \in \mathbb{C}^{n}\right\} .
\end{aligned}
$$

If we put $H_{0}=\left[\begin{array}{lll}0 & 0 & 1 \\ 0 & 0 & 0 \\ 1 & 0 & 0\end{array}\right]$, it is easy to see that $\mathfrak{a}=\mathbb{R} H_{0}$ is a maximal abelian subalgebra of $\mathfrak{p}$ and $\mathfrak{z}=\mathbb{R}\left[\begin{array}{cc}\frac{i}{n} I & 0 \\ 0 & -\mathbf{i}\end{array}\right]$ is the center of $\mathfrak{k}$, where $\mathbf{i}=\sqrt{-1}$. We have that $\mathfrak{k}=\mathfrak{k}_{s}+\mathfrak{z}$, where $\mathfrak{k}_{s}=[\mathfrak{k}, \mathfrak{k}]$ is the semisimple part of $\mathfrak{k}$. Let $M$ be the centralizer of $A$ in $K$, that is for $n>1$

$$
M=\left\{\left[\begin{array}{ccc}
e^{\mathbf{i} s} & 0 & 0 \\
0 & U & 0 \\
0 & 0 & e^{\mathbf{i} s}
\end{array}\right]: U \in \mathrm{U}(n-1), \operatorname{det}(U) e^{2 \mathbf{i} s}=1\right\} .
$$

If $\mathfrak{t}$ is the set of diagonal matrices of $\mathfrak{k}$, then $\mathfrak{t}_{c}$ is a Cartan subalgebra of $\mathfrak{g}_{c}$. The corresponding root system is

$$
\Delta=\left\{\gamma_{i, j}=\epsilon_{i}-\epsilon_{j}: 1 \leq i \neq j \leq n+1\right\}
$$

where $\varepsilon_{i}\left(\operatorname{Diag}\left(h_{1}, \ldots, h_{n+1}\right)\right)=h_{i}$. We choose an ordering in the dual space of it such that the system of positive roots is $\Delta^{+}=\left\{\gamma_{i, j}: i<j\right\}$. Let $\Delta_{c}$ 
and $\Delta_{n}$ be the set of compact and noncompact roots respectively. We fix a bilinear form $B$ on $\mathfrak{g}$, given by a multiple of the Killing form of $\mathfrak{g}$ such that $B\left(H_{0}, H_{0}\right)=1$, and for $\gamma \in \mathfrak{t}_{c}^{*}$ we denote by $H_{\gamma}$ the element of $\mathfrak{t}$ defined by $\gamma(H)=B\left(H, H_{\gamma}\right)$ for all $H \in \mathfrak{t}$. Denote by

$$
\mathfrak{t}^{-}=\mathbb{R} H_{\gamma_{1, n+1}}, \quad \text { and } \quad \mathfrak{t}^{+}=\left\{H \in \mathfrak{t}: \gamma_{1, n+1}(H)=0\right\} .
$$

Since $\left\{\gamma_{1, n+1}\right\}$ is a basis of $\Delta_{n}$, we have that $\mathfrak{t}=\mathfrak{t}^{-} \oplus \mathfrak{t}^{+}$, and there exists an automorphism $c$ of $\mathfrak{g}_{c}$, such that $c$ maps $\mathbf{i t}^{-}$bijectively to $\mathfrak{a}$, fixing $\mathfrak{t}^{+}$(see $\left[\mathbf{1 0}\right.$, p. 281]). Therefore, $\mathfrak{h}=\mathfrak{t}^{+}+\mathfrak{a}$ is a Cartan subalgebra of $\mathfrak{g}$, where

$$
\mathfrak{h}=\left\{H=\left[\begin{array}{cccc}
\mathbf{i} u_{1} & 0 & 0 & t \\
0 & \ddots & 0 & 0 \\
0 & 0 & \mathbf{i} u_{n} & 0 \\
t & 0 & 0 & \mathbf{i} u_{1}
\end{array}\right]: \begin{array}{l}
\sum_{j=2}^{n} u_{j}+2 u_{1}=0, \\
t, u_{j} \in \mathbb{R}
\end{array}\right\} .
$$

Let $\varepsilon_{j}$ be the linear funtional on $\mathfrak{a}_{c}^{*}$ defined by

$$
\varepsilon_{1}(H)=\mathbf{i} u_{1}+t, \quad \varepsilon_{n+1}(H)=\mathbf{i} u_{1}-t, \text { and } \varepsilon_{j}(H)=\mathbf{i} u_{j} \quad(1<j \leq n) .
$$

Thus, with the natural ordering, the corresponding set of positive roots is

$$
R^{+}=\left\{\alpha_{i, j}=\varepsilon_{i}-\varepsilon_{j+1}: 1 \leq i \leq j \leq n\right\} .
$$

We denote by $\Sigma$ the set of restricted roots of the pair $(\mathfrak{g}, \mathfrak{a})$, and we use a compatible ordering in the dual space of $\mathfrak{a}$. Hence, for $n>1, \Sigma^{+}=\left\{\alpha, \frac{1}{2} \alpha\right\}$ is the set of positive restricted roots, where $\alpha$ is the restriction of $\alpha_{1, n+1}$. The corresponding root spaces are given by

$$
\mathfrak{g}_{\alpha / 2}=\left\{\left[\begin{array}{ccc}
0 & { }^{t} \bar{x} & 0 \\
-x & 0 & x \\
0 & t_{\bar{x}} & 0
\end{array}\right] ; x \in \mathbb{C}^{n-1}\right\} \text { and } \mathfrak{g}_{\alpha}=\mathbb{R}\left[\begin{array}{ccc}
-\mathbf{i} & 0 & \mathbf{i} \\
0 & 0 & 0 \\
-\mathbf{i} & 0 & \mathbf{i}
\end{array}\right],
$$

and thus $m_{\alpha}=\operatorname{dim} \mathfrak{g}_{\alpha}=1$ and $m_{\alpha / 2}=\operatorname{dim} \mathfrak{g}_{\alpha / 2}=2(n-1)$.

We will identify the dual space $\mathfrak{a}_{c}^{*}$ with $\mathbb{C}$ under the correspondence $\nu=$ $z \frac{1}{2} \alpha \mapsto z$. In other words, since $\alpha\left(H_{0}\right)=2$, we are identifying $\nu$ with $\nu\left(H_{0}\right)$. As usual, let $\rho$ be the linear functional on $\mathfrak{a}$ defined by $\rho(H)=$ $\frac{1}{2} \sum_{\beta \in R^{+}} m_{\beta} \beta(H)$. Hence, under the above convention, $\rho$ is identified with $n$.

We denote by $W$ the Weyl group of $G$, and we note that in this case $W=\{ \pm \operatorname{Id}\}$.

If $A^{+}=\left\{\exp \left(t H_{0}\right): t>0\right\}$, then we have the Cartan decomposition of $G, G=K \mathrm{Cl}\left(A^{+}\right) K$. We take on $A$ the measure $d a=d t$, on $K$ we use the Haar measure so that the total mass is one, and on $G$ we use the Haar measure such that

$$
\int_{G} f(g) d g=\int_{K A^{+} K} \delta(t) f\left(k_{1} a k_{2}\right) d k_{1} d a d k_{2}
$$

where $\delta(t)=2^{2 n-1}(\sinh t)^{2(n-1)} \sinh 2 t$. 
For any $g \in G$, let $g=\kappa(g) \exp H(g) n(g)$ be the Iwasawa decomposition of $g$.

2.2. Representations. We denote by $\hat{K}$ and $\hat{M}$ the set of irreducible unitary representations of $K$ and $M$, respectively. For $l \in \mathbb{Z}$ let $\tau_{l}$ be the onedimensional representation of $K$ associated to the character $\chi_{l}\left(\left[\begin{array}{ll}A & 0 \\ 0 & y\end{array}\right]\right)=y^{l}$. We note that every one-dimensional representation of $K$ is of this form. We set $\sigma_{l}=\tau_{l \mid M}$.

For each $l \in \mathbb{Z}$, we define $m_{\alpha}(l)=1-2 l, \quad m_{\alpha / 2}(l)=2(n-1)+2 l$ and $\rho(l)=\frac{1}{2} \sum_{\beta \in R^{+}} m_{\beta}(l) \beta$. Thus, under the above identification $\rho(l)=n-l$ (see $\S 2.1)$.

Let $E_{l}$ denote the homogeneous line bundle over $G / K$ associated with $\tau_{l}$. We identify the space of $C^{\infty}$-sections of $E_{l}$ with the space $C^{\infty}\left(G / K ; \tau_{l}\right)$ of $C^{\infty}$-functions on $G$ such that $f(x k)=\tau_{l}(k)^{-1} f(x)$ for any $x \in G, k \in K$. We denote by $D_{l}=D_{l}(G / K)$, the space of left invariant differential operators on $G$ which leave $C^{\infty}\left(G / K ; \tau_{l}\right)$ invariant. We note that for $l=0, \tau_{l}$ is the trivial representation of $K$, and $D_{0}=D(G / K)$. Recall that we have the isomorphism (see for instance [12, Thm. 2.1])

$$
D_{l} \simeq U(\mathfrak{g})^{K} / U(\mathfrak{g})^{K} \cap U(\mathfrak{g}) \mathfrak{k}_{l}
$$

where $\mathfrak{k}_{l}=\left\{X+\tau_{l}(X) \mid X \in \mathfrak{z}\right\}$.

Since $\tau_{l}$ is a one-dimensional representation, $\tau_{l \mid M}$ is clearly multiplicity free (i.e., no constituent occurs twice), then by $\left[\mathbf{1}\right.$, Thm. 3] $D_{l}$ is commutative.

Definition 2.1. A complex valued function $f$ on $G$ is said to be $\tau_{l}$-radial if

$$
f\left(k_{1} x k_{2}\right)=\tau_{l}\left(k_{1}\right)^{-1} f(x) \tau_{l}\left(k_{2}\right)^{-1} \text { for all } g \in G, k_{1}, k_{2} \in K .
$$

The space of $\tau_{l}$-radial $C^{\infty}$-functions on $G$ will be denoted by $C_{l}^{\infty}(G)$. We note that $C_{l}^{\infty}(G)$ is an algebra with the following convolution product:

$$
f \star g(x)=\int_{G} f\left(y^{-1} x\right) g(y) d y .
$$

Let $f^{-}$denote the restriction to $A^{+}$of a function $f \in C_{l}^{\infty}(G)$. It follows from the Cartan decomposition $G=K \mathrm{Cl}\left(A^{+}\right) K$ that $f \in C_{l}^{\infty}(G)$ is determined by $f^{-}$. For $D \in U(\mathfrak{g})$, we denote by $\Delta_{l}(D)$ the $\tau_{l}$-radial component, that is, $\Delta_{l}(D)$ is a differential operator on $A^{+}$satisfying

$$
(D f)^{-}=\Delta_{l}(D)\left(f^{-}\right) \quad \forall f \in C_{l}^{\infty}(G) .
$$

We will now recall some facts on the radial component of $C$, the Casimir operator of $\mathfrak{g}_{c}$ with respect to $B$. Let $X_{1}, \ldots, X_{2(n-1)}$ and $X_{0}$ be basis of $\mathfrak{g}_{\alpha / 2}$ and $\mathfrak{g}_{\alpha}$ respectively, such that $-B\left(X_{i}, \theta\left(X_{j}\right)\right)=\delta_{i, j}$. Let $\left\{U_{1}, \ldots, U_{r}\right\}$ be an orthonormal basis of $\mathfrak{m}$ with respect to $-B_{\mid \mathfrak{m}}$. 
Proposition 2.2. If $f \in C_{l}^{\infty}(G)$ and $C_{\mathfrak{m}}$ denotes the Casimir element of $\mathfrak{m}$ with respect to $-B_{\mid \mathfrak{m}}$, then

$$
\begin{aligned}
& \Delta_{l}(C) f\left(a_{t}\right) \\
& =\left(\frac{d^{2}}{d t^{2}}-\tau_{l}\left(C_{\mathfrak{m}}\right)+((2 n-1) \operatorname{coth} t+2 \operatorname{coth} 2 t) \frac{d}{d t}-\frac{l^{2}}{(\cosh t)^{2}}\right) f\left(a_{t}\right) .
\end{aligned}
$$

Proof. If we define, as usually, for $j=0, \ldots, 2(n-1)$

$$
Z_{j}=2^{-\frac{1}{2}}\left(X_{j}+\theta\left(X_{j}\right)\right), \quad Y_{j}=2^{-\frac{1}{2}}\left(X_{j}-\theta\left(X_{j}\right)\right),
$$

then, it is easy to see that

$$
C=H_{0}^{2}-C_{\mathfrak{m}}+\sum_{j=0}^{2(n-1)} Y_{j}^{2}-\sum_{j=0}^{2(n-1)} Z_{j}^{2} .
$$

Using arguments analogous to those in [13, p. 280] (see also [3, Lemma 22]), we can see that in our case we obtain for $f \in C_{l}^{\infty}(G / K)$ :

$$
\begin{aligned}
C f\left(a_{t}\right)= & \frac{d^{2}}{d t^{2}} f\left(a_{t}\right)-\tau_{l}\left(C_{\mathfrak{m}}\right) f\left(a_{t}\right)+(2(n-1) \operatorname{coth} t+2 \operatorname{coth} 2 t) \frac{d}{d t} f\left(a_{t}\right) \\
& +(\sinh t)^{-2} \sum_{j=1}^{2(n-1)} \tau_{l}\left(Z_{j}^{2}\right) f\left(a_{t}\right)+(\sinh 2 t)^{-2} \tau_{l}\left(Z_{0}^{2}\right) f\left(a_{t}\right) \\
& +(\operatorname{coth} t)^{2} \sum_{j=1}^{2(n-1)} f\left(a_{t}\right) \tau_{l}\left(Z_{j}^{2}\right)+(\operatorname{coth} 2 t)^{2} f\left(a_{t}\right) \tau_{l}\left(Z_{0}^{2}\right) \\
& -2(\sinh t)^{-1}(\operatorname{coth} t) \sum_{j=1}^{2(n-1)} \tau_{l}\left(Z_{j}\right) f\left(a_{t}\right) \tau_{l}\left(Z_{j}\right) \\
& -2(\sinh 2 t)^{-1}(\operatorname{coth} 2 t) \tau_{l}\left(Z_{0}\right) f\left(a_{t}\right) \tau_{l}\left(Z_{0}\right)-\sum_{j=0}^{2(n-1)} \tau_{l}\left(Z_{j}^{2}\right) f\left(a_{t}\right) .
\end{aligned}
$$

Here, we have used that $\alpha\left(H_{0}\right)=2$, and therefore $\alpha\left(\log \left(a_{t}\right)\right)=2 t$.

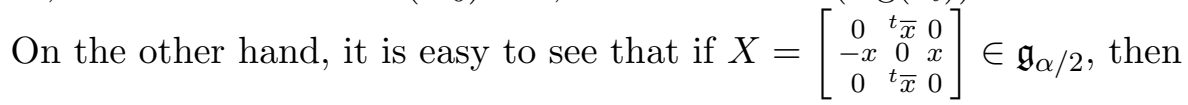

$$
(X+\theta(X))=\left[\begin{array}{crr}
0 & 2^{t} \bar{x} & 0 \\
-2 x & 0 & 0 \\
0 & & 0
\end{array}\right] \text {. }
$$

Hence, by the definition of $\tau_{l}, \tau_{l}\left(Z_{j}\right)=0$ for $j=1, \ldots, 2(n-1)$. We also have that $Z_{0}=-\mathbf{i}\left(E_{1,1}-E_{n+1, n+1}\right)$, and then $\tau_{l}\left(Z_{0}\right)=\mathbf{i} l$. Therefore, the 
above equation becomes

$$
\begin{aligned}
C f\left(a_{t}\right)= & \frac{d^{2}}{d t^{2}} f\left(a_{t}\right)-\tau_{l}\left(C_{\mathfrak{m}}\right) f\left(a_{t}\right)+(2(n-1) \operatorname{coth} t+2 \operatorname{coth} 2 t) \frac{d}{d t} f\left(a_{t}\right) \\
& -l^{2}\left((\sinh 2 t)^{-2}+(\operatorname{coth} 2 t)^{2}-2(\sinh 2 t)^{-1} \operatorname{coth} 2 t-1\right) f\left(a_{t}\right)
\end{aligned}
$$

and the last term of the right-hand side of the above equation equals $\frac{l^{2}}{(\cosh t)^{2}}$, as was to be shown.

Remark. In the case $l=0$, a $\tau_{0}$-radial function corresponds to a $K$ biinvariant function on $G$, and Proposition 2.2 generalizes the formula for the action of $\Delta(C)$ in this case given in $[8, \S 1$, p. 667$]$.

\section{Spherical functions.}

Definition 3.1. If $l \in \mathbb{Z}$ and $\phi$ is a complex valued $\tau_{l}$-radial continuous function on $G$, then $\phi$ is said to be a $\tau_{l}$-spherical function if $\phi(e)=1$ and $D \phi=\chi(D) \phi$ for each $D \in D_{l}$, with $\chi(D) \in \mathbb{C}$.

We have the following description of the $\tau_{l}$-spherical functions (see for instance [12, Prop. 6.1]), as an Einsenstein Integral (see [13, 8.12.2]).

Proposition 3.2. For $l \in \mathbb{Z}, g \in G$ and $\nu \in \mathfrak{a}^{*}$, define

$$
\Phi_{\nu, l}(g)=\int_{K} e^{-(\nu+\rho)\left(H\left(g^{-1} k\right)\right)} \tau_{l}\left(k^{-1} \kappa\left(g^{-1} k\right)\right) d k .
$$

The function $\phi_{\nu, l}$ is a $\tau_{l}$-spherical function on $G$ and every $\tau_{l}$-spherical function is of this form, for some $\nu \in \mathfrak{a}_{c}^{*}$. Furthermore, $\phi_{\nu, l}=\phi_{\mu, l}$ if and only if $\mu=s \nu$ for some $s \in W$, and the map $\nu \rightarrow \phi_{\nu, l}(g)$ is holomorphic for each $g \in G$.

In order to give another characterization of $\tau_{l}$-spherical functions, we will recall some facts on the principal series representations of $G$. Let $P=M A N$ be the minimal parabolic subgroup of $G$. For $\nu \in \mathfrak{a}_{c}^{*}$ and $l \in \mathbb{Z}$, let $\left(\pi_{\nu, l}, H_{P}^{l, \nu}\right)$ be the induced representation from $P$ to $G$ of the representation $\left(\pi^{l, \nu}, H_{\nu}\right)$ of $P$, given by $\pi^{l, \nu}(\operatorname{man}) v=a^{(\nu+\rho)} \sigma_{l}(m) v$.

Since $\left[\tau_{l}: \sigma_{l}\right]=1$, by Frobenius reciprocity, $\tau_{l}$ appears in the $K$-decomposition of $H_{P}^{l, \nu}$, and then we can define $1_{\nu, l} \in H_{P}^{l, \nu}$ such that $1_{\nu, l \mid K}=\tau_{l}$. Moreover. we have that

$$
\begin{aligned}
\left\langle\pi_{\nu, l}(g) 1_{\nu, l}, 1_{\nu, l}\right\rangle & =\int_{K} 1_{\nu, l}\left(g^{-1} k\right) \overline{1_{\nu, l}(k)} d k \\
& =\int_{K} e^{-(\nu+\rho) H\left(g^{-1} k\right)} \tau_{-l}\left(k^{-1} \kappa\left(g^{-1} k\right)\right) d k
\end{aligned}
$$


This means that $\phi_{\nu,-l}(g)=\left\langle\pi_{\nu, l}(g) 1_{\nu, l}, 1_{\nu, l}\right\rangle$, and so it can be shown that the restriction $\Phi_{\nu, l}^{-}$of $\Phi_{\nu, l}$ to $A^{+}$satisfies the differential equation:

$$
\Delta_{l}(C) \Phi_{\nu, l}^{-}=\chi(\nu, l) \Phi_{\nu, l}^{-}
$$

where $\chi(\nu, l)=\nu^{2}-\rho^{2}+\tau_{-l}\left(C_{\mathfrak{m}}\right)([13$, p. 280]).

As in the case of the trivial $K$-type, these spherical functions are also related with hypergeometric functions, as we will now see.

Proposition 3.3 ([11, Prop. 2.6]). Let $u(t)=2 \cosh t$, then we have that

$$
u(t)^{l} \cdot\left(\Delta_{l}(C)+\rho^{2}-\tau_{l}\left(C_{\mathfrak{m}}\right)\right) \cdot u(t)^{-l}=L(l)+\rho(l)^{2}
$$

where $L(l)=\frac{d^{2}}{d t^{2}}+((2 n-1) \operatorname{coth} t+(1-2 l) \tanh t) \frac{d}{d t}$.

Using this proposition one can see that the function

$$
\psi(t)=u^{l}(t) \Phi_{\nu, l}^{-}\left(\exp \left(t H_{0}\right)\right)
$$

is an even smooth function on $(0,+\infty)$ satisfying $\psi(0)=1$ and

$$
L(l) \psi=\lambda(\nu, l) \psi
$$

where $\lambda(\nu, l)=\nu^{2}-\rho(l)^{2}$. Furthermore, it is known that the Jacobi function

$$
\phi_{i \nu}^{(n-1,-l)}={ }_{2} F_{1}\left(\frac{n-l+\nu}{2}, \frac{n-l-\nu}{2}, n,-(\sinh t)^{2}\right)
$$

is the unique solution satisfying these conditions (see $[\mathbf{6}, \S 2.1])$. Therefore

$$
\Phi_{\nu, l}\left(\exp t H_{0}\right)=(2 \cosh t)^{-l} \phi_{i \nu}^{(n-1,-l)}(t) .
$$

It can also be seen (see $[\mathbf{6}$, p. 7] and $[\mathbf{1 1}$, p. 384]) that for $\nu \notin-\mathbb{N}$, a second solution of $(1)$ in $(0,+\infty)$ is given by

$$
\widetilde{Q}_{\nu, l}(t)=(2 \cosh t)^{-(\nu+\rho(l))}{ }_{2} F_{1}\left(\frac{n-l+\nu}{2}, \frac{n+l+\nu}{2}, 1+\nu,(\cosh t)^{-2}\right) .
$$

As a function of $\nu, \widetilde{Q}_{\nu, l}$ is holomorphic on $\mathbb{C} \backslash \mathbb{N}$, and for $\nu \notin \mathbb{Z}, \widetilde{Q}_{\nu, l}$ and $\widetilde{Q}_{-\nu, l}$ are linearly independent, and so, as in the case $l=0$, we can write

$$
(2 \cosh t)^{l} \Phi_{\nu, l}\left(\exp \left(t H_{0}\right)\right)=c(\nu, l) \widetilde{Q}_{-\nu, l}(t)+c(-\nu, l) \widetilde{Q}_{\nu, l}(t)
$$

where

$$
c(\nu, l)=\frac{2^{n-l-\nu}(n-1) ! \Gamma(\nu)}{\Gamma\left(\frac{\nu+n+l}{2}\right) \Gamma\left(\frac{\nu+n-l}{2}\right)},
$$

and if $\operatorname{Re} \nu>0$, the asymptotic behavior of $\Phi_{\nu, l}$, as $t \rightarrow \infty$, is given by:

$$
\Phi_{\nu, l}\left(\exp \left(t H_{0}\right)\right) \sim c(\nu, l) e^{t(\nu-\rho)} .
$$


We will also need the following fact (see [4, Prop. 2.2]):

(6)

$$
\begin{aligned}
& \delta^{1 / 2} \cdot\left(\Delta_{l}(C)+\rho^{2}\right) \cdot \delta^{-1 / 2} \\
& =\frac{d^{2}}{d t^{2}}+\tau_{-l}\left(C_{\mathfrak{m}}\right)+\sum_{\beta \in R^{+}} \frac{1}{4} m_{\frac{\beta}{2}}(l)\left(2-m_{\frac{\beta}{2}}(l)-2 m_{\beta}(l)\right) 4 \sinh (2 t)^{-2} .
\end{aligned}
$$

With all these elements in place, we can adapt most of the arguments in $[\mathbf{8}, \mathbf{9}]$, to obtain generalizations of the results of $[\mathbf{9}, \S 3]$.

Theorem 3.4. If $\nu \in \mathbf{C}, \nu \notin-\mathbb{N}$, then there exists a function $Q_{\nu, l} \in$ $C_{-l}^{\infty}(G-K)$ with the following properties:

(a) $\Delta_{l}(C) Q_{\nu, l}=\chi(\nu, l) Q_{\nu, l} . Q_{\nu, l}(x)$ is holomorphic for $\nu \notin-\mathbb{N}$ and if $\nu \in-\mathbb{N}, Q_{\nu, l}(x)$ has at most a simple pole.

(b) $\Phi_{\nu, l}^{-}=c(-\nu, l) Q_{\nu, l}^{-}+c(\nu, l) Q_{-\nu, l}^{-}$.

(c) Ast $\mapsto 0, Q_{\nu, l}\left(\exp \left(t H_{0}\right)\right) \sim d(\nu) t^{-2(n-1)}|\log t|^{\delta_{n, 1}}$, for some meromorphic function $d(\nu)$ on $\mathbb{C}$, holomorphic if $\nu \notin-\mathbb{N}$. Furthermore, if $\nu \in$ $\mathbb{C} \backslash-\mathbb{N}$, then $Q_{\nu, l}(g)$ lies in $L_{\mathrm{loc}}^{1}(G)$, and if $\operatorname{Re} \nu>\rho, Q_{\nu, l}(g) \in L^{1}(G)$.

(d) $\lim _{t \mapsto 0^{+}} \delta(t) \frac{d}{d t} Q_{\nu, l}\left(\exp \left(t H_{0}\right)\right)=-2 \nu c(\nu, l)$.

(e) If $f \in C_{c}^{\infty}\left(G / K, \tau_{l}\right)$ and $\nu \notin-\mathbb{N}$ then for $\operatorname{Re} \nu>\rho$

$$
\int_{G} Q_{\nu, l}\left(x^{-1} y\right)(C-\lambda(\nu, l) \mathrm{Id}) \mathrm{f}(\mathrm{y}) \mathrm{dy}=-2 \nu c(\nu, l) f(x) .
$$

Proof. Let $Q_{\nu, l}\left(k a_{t} k^{\prime}\right)=\tau_{l}(k) u(t)^{-l} \widetilde{Q}_{\nu, l}(t) \tau_{l}\left(k^{\prime}\right)$. As we noted before, since $\widetilde{Q}_{\nu, l}$ is a solution of $L(l) g(t)=\lambda(\nu, l) g(t), Q_{\nu, l}$ is a solution of $\Delta_{l}(C) f^{-}=$ $\chi(\nu, l) f^{-}$.

It is clear from the definition that $Q_{\nu, l} \in C_{-l}^{\infty}(G \backslash K)$, and by the above observations, it satisfies (a). From our definition, it is also clear that (b) is equivalent to (3).

The proof of (c) is similar to that in the case of the trivial $K$-type, so it will be omitted (see [9]). Note that $(2)$ implies that $\widetilde{Q}_{\nu, l}(t) \sim e^{-t(\nu+\rho(l))}$ when $t \mapsto \infty$, therefore $Q_{\nu, l}\left(\exp \left(t H_{0}\right)\right) \sim e^{-(\nu+\rho)}$ when $t \mapsto \infty$. This fact allows us to prove (d) as in the case of $l=0$.

In order to see (e), we first note that since if $f \in C_{c}^{\infty}\left(G / K, \tau_{l}\right)$, so is $L_{x^{-1}} f$, then it suffices to see that

$$
\int_{G} Q_{\nu, l}(y)(C-\lambda(\nu, l)) f(y) d y=-2 \nu c(\nu, l) f(e) .
$$

The left-hand side equals

$$
\int_{0}^{\infty} Q_{\nu, l}\left(a_{t}\right)(C-\lambda(\nu, l)) \int_{K} \tau_{l}(k) f\left(k a_{t}\right) d k \delta(t) d t .
$$


If $f \in C_{c}^{\infty}\left(G / K, \tau_{l}\right)$, then $f^{l}\left(a_{t}\right):=\int_{K} \tau_{l}(k) f\left(k a_{t}\right) d k$ is a $\tau_{l}$-radial function on $G$. Hence we can replace $C$ by its radial part to obtain

$$
\int_{0}^{\infty}\left(\delta^{\frac{1}{2}}(t) Q_{\nu, l}\left(a_{t}\right) \delta^{\frac{1}{2}}(t) \Delta_{l}(C) f^{l}\left(a_{t}\right)-\delta^{\frac{1}{2}}(t) \Delta_{l}(C) Q_{\nu, l}\left(a_{t}\right) \delta^{\frac{1}{2}}(t) f^{l}\left(a_{t}\right)\right) d t .
$$

Now using the radialization (6) and arguing as in [9, pp. 1225], we obtain that the above is equal to

$$
\int_{0}^{\infty} \frac{d}{d t}\left(\delta(t) Q_{\nu, l}\left(\exp \left(t H_{0}\right)\right) \frac{d}{d t} f^{l}\left(a_{t}\right)-\delta(t) \frac{d}{d t} Q_{\nu, l}\left(\exp \left(t H_{0}\right)\right) f^{l}\left(a_{t}\right)\right) d t .
$$

Therefore, looking at the asymptotic behavior as $t \mapsto 0$, and as $t \mapsto \infty$, we obtain that the above integral equals $\lim _{t \mapsto 0^{+}} \delta(t) \frac{d}{d t} Q_{\nu, l}\left(\exp \left(t H_{0}\right)\right) f^{l}(e)$. Then, using (d) we are done.

\section{The residues of the resolvent kernel.}

Let $\widetilde{R}(\lambda(\nu, l))$ denote the kernel operator with kernel $K_{\nu, l}(x, y):=-\frac{Q_{\nu, l}\left(x^{-1} y\right)}{2 \nu c(\nu, l)}$ and let $R(\lambda(\nu, l))$ denote the resolvent of $C$ acting on $L^{2}\left(G / K, \tau_{l}\right)$. By Theorem 3.4, if $\operatorname{Re} \nu>\rho$, then $\widetilde{R}(\lambda(\nu, l))=R(\lambda(\nu, l))$.

Since $\nu \mapsto K_{\nu, l}$ is defined also for $\operatorname{Re} \nu \leq \rho$, we are interested in $\widetilde{R}(\lambda(\nu, l))$ acting on $C_{c}^{\infty}\left(G / K, \tau_{l}\right)$ as a meromorphic continuation of $R(\lambda(\nu, l))$. In the next theorem, we will give a description of the singularities of $\widetilde{R}(\lambda(\nu, l))$.

Theorem 4.1. $\widetilde{R}(\lambda(\nu, l))$ has simple poles lying at $\nu=\nu_{k, l}^{ \pm}$with $\nu_{k, l}^{-}=$ $-|l|-n-2 k, k \in \mathbb{N}_{0}$, and $\nu_{k, l}^{+}=|l|-n-2 k$, for $k \in \mathbb{N}_{0}$ such that $|l|-n-2 k \geq 0$. If $\nu$ is a pole and we set, for $f \in C_{c}^{\infty}\left(G, K, \tau_{l}\right), T_{\nu}(f):=$ $\operatorname{Res}_{z=\nu} \widetilde{R}(\lambda(z, l))(f)$, then $T_{\nu}(f)=p(\nu) f * \check{\Phi}_{\nu,-l}$.

Proof. We know that $\Phi_{\nu, l}(g)$ is everywhere holomorphic as a function of $\nu$. Hence, using (a) and (b) of Theorem 3.4, we find that the poles of $K_{\nu, l}$ are precisely the zeros of $2 \nu c(\nu, l)$.

Furthermore, it is easy to see from (4) that the zeros of $c(\nu, l)$ are at $\nu=\nu_{k, l}^{ \pm}$, as in the statement of the theorem. On the other hand, we have that $\left|\nu_{k, l}^{+}\right|<\left|\nu_{0, l}^{-}\right|$, when $|l|>n$, so that $\nu_{k, l}^{+}$is defined. Hence $\frac{Q_{-\nu, l}}{2 \nu c(-\nu, l)}$ is analytic at $\nu_{k, l}^{ \pm}$. Thus, for $f \in C_{c}^{\infty}\left(G / K, \tau_{l}\right)$, using Theorem 3.4(b), we get that if $\nu$ is a pole, then

$$
\operatorname{Res}_{z=\nu} \widetilde{R}(\lambda(z, l))(f)=p(\nu) f * \check{\Phi}_{\nu,-l},
$$

where $p(\nu)=-\operatorname{Res}_{z=\nu}(2 \nu c(\nu, l) c(-\nu, l))^{-1}$.

Now we want to study the image of these operators, and in order to do this, we will introduce certain irreducible representations of $K$, for $n>1$. 
For $p, q \in \mathbb{N}_{0}$, we denote by $V_{p, q}$, the set of harmonic polynomials in $z \in \mathbb{C}^{n}$ of bidegree $(p, q)$, and define on this space the action of $K$ given by

$$
\tau_{l, p, q}\left(\left[\begin{array}{cc}
A & 0 \\
0 & y
\end{array}\right]\right) f(z)=y^{q-p+l} f\left({ }^{t} z A\right) .
$$

Proposition $4.2([\mathbf{2}, \S 2])$. Let $\tau$ be an arbitrary $K$-type which contains the $M$-type $\sigma_{l}$. Then there exist $p, q \in \mathbb{N}_{0}$ such that $\tau$ is equivalent with $V_{p, q}$.

Actually, if we put $F_{p, q}(z)=z_{1}^{p} \bar{z}_{1}^{q}{ }_{2} F_{1}\left(-p,-q, n-1,-\left(\left|z_{2}\right|^{2}+\cdots+\right.\right.$ $\left.\left|z_{n}\right|^{2}\right) /\left|z_{1}\right|^{2}$ ) then $F_{p, q} \in V_{p, q}$, and it is easy to see that $\tau_{l, p, q}(X) F_{p, q}=$ $\sigma_{l}(X) F_{p, q}$ for $X \in M$.

For any $P \in \operatorname{Hom}_{M}\left(V_{p, q}, H_{l}\right)$ and $\nu \in \mathfrak{a}_{c}^{*}$ we can define a $K$-intertwining operator from $V_{p, q}$ to $H_{P}^{l, \nu}$ by $L(P, f, \nu)(g)=e^{-(\nu+\rho) H(g)} P\left(\tau_{l, p, q}\left(\kappa(g)^{-1}\right) f\right)$, for $f \in V_{p, q}$ (see $\left.[\mathbf{1 3}, 8.11 .4]\right)$. Furthermore, since $\left[\tau_{l, p, q}: \sigma_{l}\right]=1$, we have that $f \mapsto L(P, f, \nu)$ is an injective $K$-intertwining operator.

Let $P$ denote the linear map from $V_{p, q}$ to $\mathbb{C}$ defined by $P(f)=f(1,0, \ldots$, $0)$. It is clear that $P \in \operatorname{Hom}_{M}\left(V_{p, q}, H_{l}\right)$, and then we have the related homomorphism $L(P, f, \nu)$. Let $\widetilde{V}_{p, q} \subset H_{P}^{l, \nu}$ denote the image of $V_{p, q}$ under this homomorphism and let $A(w, l, \nu): H_{P}^{\nu, l} \mapsto H_{P}^{-\nu, l}$ denote the standard intertwining operator, where $w=\operatorname{diag}(-1,-1,1, \ldots, 1) \in K$ is a representative of the nontrivial element of $W$.

In particular, from $[\mathbf{2}, \S 3]$ we have that

$$
A(w, l, \nu) L\left(P, F_{p, q}, \nu\right)=(-1)^{p+q} c_{\tau_{l, p, q}}\left(\sigma_{l}, \nu\right) L\left(P, F_{p, q},-\nu\right)
$$

where $c_{\tau_{l, p, q}}\left(\sigma_{l}, \nu\right)$ is given by

$$
c_{\tau_{l, p, q}}\left(\sigma_{l}, \nu\right)=\frac{k \Gamma(\nu) \prod_{j=0}^{p-1}(\nu-n+l-2 j) \prod_{j=0}^{q-1}(\nu-n-l-2 j)}{\Gamma\left(\frac{\nu+n-l+2 p}{2}\right) \Gamma\left(\frac{\nu+n+l+2 q}{2}\right)} .
$$

If $\nu \neq 0$, let $D_{\nu}^{l}=\left\{(p, q) \in \mathbb{N}_{0}^{2}: c_{\tau_{l, p, q}}\left(\sigma_{l}, \nu\right)=0\right\}$. If $(p, q) \in D_{\nu}^{l}$, it is clear that $L\left(P, F_{p, q}, \nu\right) \in \operatorname{Ker} A(w, l, \nu)$ which is a $G$-module. Hence $\widetilde{V}_{p, q} \subset$ $\operatorname{Ker} A(w, l, \nu)$, and moreover, by Frobenius reciprocity and Proposition 4.2 we have that

$$
\operatorname{Ker} A(w, l, \nu)=\oplus_{(p, q) \in D_{\nu}^{l}} \widetilde{V}_{p, q} .
$$

It is easy to see that

$$
D_{\nu_{k}^{-}}^{l}=\left\{(p, q) \in \mathbb{N}_{0}^{2}: p \leq k+\frac{l+|l|}{2}, q \leq k+\frac{|l|-l}{2}\right\},
$$

and therefore $\operatorname{Ker} A\left(w, l, \nu_{k}^{-}\right)=\sum_{(p, q) \in D_{\nu_{k}^{-}}^{l}} \widetilde{V}_{p, q}$ is a finite dimensional $(\mathfrak{g}, K)$ module. It is clear that its restriction contains $\tau_{l}=\tau_{l, 0,0}$.

For $\nu=0$, since we know that $c(\nu, l)$ has a pole, we can consider the normalized intertwining operator $B(w, l, \nu)=\Gamma(\nu)^{-1} A(w, l, \nu)$; now, since 
$\Gamma(\nu)^{-1} c_{\tau_{l, p, q}}\left(\sigma_{l}, \nu\right)$ is holomorphic at $\nu=0$, then as in the other cases, we have that $\operatorname{Ker} B(w, l, 0)=\sum_{(p, q) \in D_{0}^{l}} \widetilde{V}_{p, q}$, where

$$
D_{0}^{l}=\left\{(p, q) \in \mathbb{N}_{0}^{2}:\left.\Gamma(\nu)^{-1} c_{\tau_{l, p, q}}\left(\sigma_{l}, \nu\right)\right|_{\nu=0}=0\right\} .
$$

We note that since $\nu=0$ is a pole, then $|l|-n=2 k$ with $k \in \mathbb{N}$ and one can verifies that

$$
\begin{aligned}
& D_{0}^{l}=\left\{(p, q) \in \mathbb{N}_{0}^{2}: p \leq k=\frac{l-n}{2}\right\}, \quad \text { if } l>0, \\
& D_{0}^{l}=\left\{(p, q) \in \mathbb{N}_{0}^{2}: q \leq k=\frac{-l-n}{2}\right\}, \quad \text { if } l<0 .
\end{aligned}
$$

It is well-known that $H_{P}^{l, \nu}$ is equivalent to $H_{\bar{P}}^{l,-\nu}$ and the intertwining operator is $R(w)$, where $R$ is the right regular representation of $G$. It is also known that if $A\left(\bar{P}, P, \sigma_{l}, \nu\right)$ denotes the standard intertwining operator from $H_{P}^{l, \nu}$ to $H_{\bar{P}}^{l, \nu}$, then $A(w, l, \nu)=R(w) A\left(\bar{P}, P, \sigma_{l}, \nu\right)$ (see [5, VII $\left.\S 4\right]$ ).

Let $V(\mu, l)$ denote the image of the residue of $\widetilde{R}(\lambda(\nu, l))$ at $\nu=\mu$, and $V(\mu, l)_{K}$ the space of $K$-finite vectors in $V(\mu, l)$. Now, using a generalization of Helgason's theorem $([\mathbf{1 0}, \S \mathbf{7}])$, we can give a very explicit description of $V(\mu, l)_{K}$.

Theorem 4.3. If $\mu$ is a pole of $\widetilde{R}(\lambda(\nu, l))$, then $V(\mu, l)_{K}$ is a $(\mathfrak{g}, K)$-module. This module is of finite dimension only in the case when $\mu=\nu_{k, l}^{-}$for $k \in \mathbb{N}_{0}$. The modules corresponding to $\mu=\nu_{k, l}^{+}$are equivalent, as $(\mathfrak{g}, K)$-modules, to holomorphic discrete series representations. Moreover, in the case when $\mu=\nu_{k, l}^{-}$and $\mu=0$ these $(\mathfrak{g}, K)$-modules are isomorphic to $\operatorname{Ker} A\left(w, l, \nu_{k, l}^{-}\right)$ and $\operatorname{Ker} B(w, l, 0)$ respectively.

Proof. If $f \in C_{c}^{\infty}(G / K, l)$ and $x \in G$, by Theorem 4.1 we have that:

$$
\begin{aligned}
T_{\nu_{k, l}^{ \pm}}(f)(x) & =p\left(\nu_{k, l}^{ \pm}\right) f * \check{\phi}_{\nu_{k, l}^{ \pm},-l}(x) \\
& =p\left(\nu_{k, l}^{ \pm}\right)\left\langle\pi_{\nu_{k, l}^{ \pm}}\left(x^{-1}\right) \pi_{\nu_{k, l}^{ \pm}, l}(f) 1_{\nu_{k, l}^{ \pm}, l}, 1_{\nu_{k, l}^{ \pm}, l}\right\rangle .
\end{aligned}
$$

Hence, $V(\nu, l)_{K}$ is isomorphic to the $(\mathfrak{g}, K)$-module generated by $1_{l, \nu}$, so we will now describe this module.

In order to do this, we will give a condition on $\nu$ for $1_{l, \nu}$ to generate a finite dimensional $(\mathfrak{g}, K)$-submodule of $H_{P}^{l, \nu}$.

For $\lambda \in \mathfrak{h}_{c}^{*}$ we define

$$
m_{0}=\lambda(\mathbf{i} X) \text { and } m_{1}=\frac{2\langle\lambda, \alpha\rangle}{\langle\alpha, \alpha\rangle},
$$


where as in $[\mathbf{1 0}, 4.4], X=\left[\begin{array}{lll}-1 & & \\ & \frac{2}{n-1} \mathrm{Id} & \\ & & -1\end{array}\right]$.

If $\left.\lambda\right|_{\mathfrak{t}^{+} \cap \mathfrak{k}_{1}}=0$, then by [10, Prop 7.1], $\lambda$ is dominant integral if and only if $m_{0}$ and $m_{1}$ are integers such that $\left|m_{0}\right| \leq m_{1}$ and $(-1)^{m_{0}}=(-1)^{m_{1}}$.

We note that $\left\{\alpha, \varepsilon_{1}, \ldots, \varepsilon_{n}\right\}$ is a basis of $\mathfrak{h}_{c}^{*}$, then straightforward calculation shows that if $\lambda=a_{1} \alpha+\sum_{i=2}^{n} a_{i} \varepsilon_{i} \in \mathfrak{h}_{c}^{*}$, then $\left.\lambda\right|_{\mathfrak{t}^{+} \cap \mathfrak{k}_{1}}=0$ if and only if $a_{2}=a_{3}=\cdots=a_{n}$. Hence, if we denote $\beta=\sum_{i=2}^{n} \varepsilon_{i}$, and $\lambda=a_{1} \alpha+a_{0} \beta$, then we have that $m_{0}=-2 a_{0}$ and $m_{1}=2 a_{1}$.

On the other hand, by [10, Thm. 7.2], $\lambda=a_{1} \alpha+a_{0} \beta$, is a highest weight of a finite dimensional irreducible representation of $G$, whose restriction to $K$ contains the one-dimensional $K$-type $\chi_{m_{0}}$ with multiplicity one.

Furthermore, in the proof of the theorem, we can see that this representation is equivalent to $\operatorname{Im} A\left(\bar{P}, P, \sigma_{l}, \mu\right)$, a subrepresentation of $H_{\bar{P}}^{l, \nu}$, where $\mu=\lambda_{\mid \mathfrak{a}}+\rho$.

Therefore, $1_{l, \nu}$ generates a finite dimensional $G$-submodule of $H_{P}^{l, \nu}$ (with highest weight $\lambda$ ) if and only if $\nu=-\lambda_{\mid \mathfrak{a}}-\rho$, where $\lambda=\frac{l}{2} \beta+(|l|+2 k) \frac{\alpha}{2}$.

We note that because of our identification, $\nu=\nu_{k, l}$, as we want to show.

On the other hand, we have proved that $\operatorname{Ker} A\left(w, l, \nu_{k, l}^{-}\right)$is a finite dimensional submodule of $H_{P}^{l, \nu_{k, l}^{-}}$which contains $1_{l, \nu_{k, l}^{-}}$. We also know that $H_{P}^{l, \nu_{k, l}^{-}}$ is equivalent to $H_{\bar{P}}^{l,-\nu_{k, l}^{-}}$, and it has only one irreducible representation ([5, p. 273]). Therefore, it is clear that the $(\mathfrak{g}, K)$-submodule of $H_{P}^{l, \nu_{k, l}^{-}}$generated by $1_{l, \nu_{k, l}^{-}}$is $\operatorname{Ker} A\left(w, l, \nu_{k, l}^{-}\right)$.

We will now study the case when $\nu=\nu_{k, l}^{+}$. We begin by observing that in the rank one case, $\left[\mathbf{1 1}\right.$, Thm. 5.1] states that if $\lambda \in \mathfrak{a}_{c}^{*}, \lambda=\nu \cdot \frac{\alpha}{2}$, with $\nu \geq 0$, then $\Phi_{\lambda, l}$ belongs to $L^{2}\left(G / K, \tau_{l}\right)$ if and only if

$$
\frac{\langle-\lambda-\rho(|l|), \alpha\rangle}{\langle\alpha, \alpha\rangle} \in \mathbb{N} .
$$

In particular, there exists $\lambda \in \mathfrak{a}_{c}^{*}$ such that $\Phi_{\lambda, l}$ belongs to $L^{2}\left(G / K, \tau_{l}\right)$ if and only if $|l|>n$. We note that (8) means that $-\nu-(n-|l|)=2 k$ with $k \in \mathbb{N}$, or equivalently, $\nu=\nu_{k, l}^{+}$for some $k$. Hence the $(\mathfrak{g}, K)$-module generated by $1_{l, \nu}$ in $H_{P}^{l, \nu}$ is infinitesimally equivalent to a discrete series representation if and only if $\nu=\nu_{k, l}^{+}$

Moreover, Shimeno proves in [11, Thm 5.10], that these are actually infinitesimally equivalent to holomorphic discrete series representations.

For $\nu=0$, it is known that if $H_{p}^{l, 0}$ is reducible, it is a sum of two inequivalent irreducible representations. These representations are called limits of discrete series. Since they are inequivalent and $B(w, l, 0)$ is an intertwining 
operator, it is easy to see that $\operatorname{Ker} B(w, l, 0)$ is the $(\mathfrak{g}, K)$-submodule of $H_{P}^{l, 0}$ generated by $1_{l, 0}$, concluding the proof.

Remark 4.4. We wish to point out that every (irreducible) finite dimensional, discrete series, or limit of discrete series representation of $G$, containing a one-dimensional $K$-type can be seen as a residue of the resolvent kernel. That is, if $\left(\pi, H_{\pi}\right)$ is a finite dimensional representation of $G$ containing a one-dimensional $K$-type $\chi_{m}$, then there exists a line bundle over $G / K$ such that this representation is isomorphic to the residue of the meromorphic continuation of the resolvent of the Casimir operator acting on that line bundle. In fact, if $\lambda$ is the highest weight of $\pi$, then by [10, Thm 7.2] $\lambda=a \alpha+b \beta$, where $a=|b|+k$. Then by the above, $H_{\pi}$ is isomorphic to $V_{k, 2 b}$, the image of the residue of $R(\lambda(\nu, 2 b))$ at $\nu=\nu_{k, 2 b}^{-}$.

In the case that $\left(\pi, H_{\pi}\right)$ is a discrete series, this implies that $\Phi_{\lambda, m}$ belongs to $L^{2}\left(G / K, \tau_{m}\right)$, and then by [11, Thm 5.1] $\lambda=\nu_{k, m}^{+}$. Hence, $H_{\pi}$ is isomorphic to $V\left(\nu_{k, m}^{+}, m\right)$.

Finally, if $\left(\pi, H_{\pi}\right)$ is a limit of discrete series containing the one-dimensional $K$-type $\tau_{m}$, it means that $H_{P}^{m, 0}$ is reducible, and so $m \equiv n \quad(2)$ and $|m|>n\left([\mathbf{5}\right.$, p. 621] $)$. Thus, $H_{\pi}$ is isomorphic to the image of the residue of $R(\lambda(\nu, m))$ at $\nu=\nu_{\frac{|m|-n}{2}, m}^{+}$.

Remark 4.5. We observe that $\nu=0$ is not a pole of the resolvent kernel $\widetilde{R}_{l}(\lambda(\nu, l))$, in the case when $l=0$.

We will now use the Weyl dimension formula to calculate the dimension of the representation $V\left(\nu_{k, l}^{-}\right)$. The fundamental weights of $\mathfrak{g}_{c}$ are $\Lambda_{j}=$ $\epsilon_{1}+\cdots+\epsilon_{j}, 1 \leq j \leq n$, hence $\alpha=\Lambda_{1}+\Lambda_{n}$ and $\beta=\Lambda_{n}-\Lambda_{1}$. Hence, we are interested in the dimension of the $\mathfrak{g}_{c}$-module asociated to $\Lambda_{k, l}=$ $\frac{l}{2} \beta+\left(\frac{|l|+2 k}{2}\right) \alpha=\left(\frac{|l|-l}{2}+k\right) \Lambda_{1}+\left(\frac{|l|+l}{2}+k\right) \Lambda_{n}$. Then we have that

$$
\begin{aligned}
\operatorname{dim}\left(V_{k, l}\right)= & \prod_{1 \leq i<j \leq n+1} \frac{\left\langle\Lambda_{k, l}+\rho, \epsilon_{i}-\epsilon_{j}\right\rangle}{\left\langle\rho, \epsilon_{i}-\epsilon_{j}\right\rangle} \\
= & \prod_{1<j \leq n} \frac{\frac{|l|-l}{2}+k+j-1}{j-1} \cdot \prod_{1<i \leq n} \frac{\frac{|l|+l}{2}+k+n+1-i}{n+1-i} \\
& \cdot \frac{1}{n}\left(\frac{|l|+l}{2}+\frac{|l|-l}{2}+2 k+n\right)
\end{aligned}
$$

and so

$$
\operatorname{dim}\left(V_{k, l}\right)=\left(\begin{array}{c}
\frac{|l|-l}{2}+k+n-1 \\
\frac{|l|-l}{2}+k
\end{array}\right) \cdot\left(\begin{array}{c}
\frac{|l|+l}{2}+k+n-1 \\
\frac{|l|+l}{2}+k
\end{array}\right) \cdot \frac{|l|+2 k+n}{n} .
$$




\section{The case $G=\mathrm{S} U(1,1)$.}

We will consider now the case when $G=\mathrm{S} U(1,1)$. We shall see that the results will be entirely similar to those in the case of $\mathrm{S} U(n, 1), n>1$, but we shall analyze this case separately, because the notation and some of the definitions are different.

We have that $\theta(X)=\bar{X}^{t}$ and $\mathfrak{g}=\mathfrak{k}+\mathfrak{p}$, where

$$
\mathfrak{k}=\left\{\left[\begin{array}{cc}
\mathbf{i} t & 0 \\
0 & -\mathbf{i} t
\end{array}\right]: t \in \mathbb{R}\right\} \text { and } \mathfrak{p}=\left\{\left[\begin{array}{cc}
0 & b \\
\bar{b} & 0
\end{array}\right]: b \in \mathbb{C}\right\} \text {. }
$$

If $H_{0}=\left[\begin{array}{ll}0 & 1 \\ 1 & 0\end{array}\right]$ and $\mathfrak{a}=\mathbb{R} H_{0}$, then $M=\{ \pm I\}$ and in this case, $\hat{K}=$ $\left\{\tau_{l}: l \in \mathbb{Z}\right\}$ and $\hat{M}=\{1, \epsilon\}$, where $\epsilon$ denotes the nontrivial character of $M$. Therefore, for each $\nu \in \mathbb{C}$ we have two principal series representations, $H^{\nu,+}$ and $H^{\nu,-}$ corresponding to 1 and $\epsilon$, respectively and $\tau_{l \mid \mathfrak{m}}=I$ if and only if $l \equiv 0(2), \tau_{l \mid \mathfrak{m}}=\epsilon$, otherwise. Now, Proposition 2.2 may be stated as follows:

$$
\Delta_{l}(C)=\frac{d^{2}}{d t^{2}}+2 \operatorname{coth} t \frac{d}{d t}+l^{2}(\cosh t)^{-2} .
$$

Furthermore, Proposition 3.3 becomes

$$
u(t)^{l} \circ\left(\Delta_{l}(C)+\rho^{2}\right) \circ u(t)^{-l}=\frac{d^{2}}{d t^{2}}+(\operatorname{coth} t+(1-2 l) \tanh t) \frac{d}{d t}+\rho(l)^{2},
$$

where $u(t)=2 \cosh t$.

We now define a differential operator on $\mathbb{R}^{+}$, as in Proposition 3.3:

$$
L(l)=\frac{d^{2}}{d t^{2}}+(\operatorname{coth}(t)+(1-2 l) \tanh (t)) \frac{d}{d t} .
$$

As in the case when $n>1$, one can relate the spherical functions $\Phi_{\nu, l}$ with the solutions of $L(l) f=\left(\nu^{2}-\rho(l)^{2}\right) f$, where $\rho(l)=1-l$ (see $\S 2.1$ ), and find that they are given by

$$
\Phi_{\nu, l}\left(\exp \left(t H_{0}\right)\right)=(2 \cosh t)^{-l} \phi_{i \nu}^{(0,-l)}(t) .
$$

In the same way, we can see that if we take the solution in (2) of the above equation for $n=1$ and $\nu \notin-\mathbb{N}$, we get the following eigenfunction of $\Delta_{l}(C)$ on $A^{+}$:

$$
\begin{aligned}
& Q_{\nu, l}\left(\exp t H_{0}\right) \\
& =(2 \cosh (t))^{-(\nu+\rho(l))}{ }_{2} F_{1}\left(\frac{1-l+\nu}{2}, \frac{1+l+\nu}{2}, 1+\nu, \cosh (t)^{-2}\right),
\end{aligned}
$$

which satisfies

$$
(2 \cosh t)^{l} \Phi_{\nu, l}\left(\exp \left(t H_{0}\right)\right)=c(\nu, l) Q_{-\nu, l}\left(a_{t}\right)+c(-\nu, l) Q_{\nu, l}\left(a_{t}\right)
$$


where

$$
c(\nu, l)=\frac{2^{1-l-\nu} \Gamma(\nu)}{\Gamma\left(\frac{\nu+1+l}{2}\right) \Gamma\left(\frac{\nu+1-l}{2}\right)} .
$$

With all this in place, we can prove Theorem 3.4 in our case, obtaining in the same way the meromorphic continuation of the resolvent kernel $K_{\nu, l}(x, y)=-\frac{Q_{\nu, l}\left(x^{-1} y\right)}{2 \nu c(\nu, l)}$, hence we have the following theorem, which gives results analogous to those in Theorem 4.1 and Theorem 4.3 in the present case.

Theorem 5.1. $\widetilde{R}(\lambda(\nu))$ has simple poles lying at $\nu=\nu_{k, l}^{ \pm}$, where $\nu_{k, l}^{-}=$ $-|l|-1-2 k$, with $k \in \mathbb{N}_{0}$, and $\nu_{k, l}^{+}=|l|-1-2 k$, with $k \in \mathbb{N}$ such that $|l|-1-2 k \geq 0$. If $\nu$ is a pole, $\operatorname{Res}_{z=\nu} \widetilde{R}(\lambda(z))(f)=p(\nu) f * \check{\phi}_{\nu,-l}$.

Moreover, the rank of the residue of $\widetilde{R}(\lambda(\nu))$ at $\nu=\nu_{k, l}^{-}$is a finite dimensional $(\mathfrak{g}, K)$-module. The residues at $\nu=\nu_{k, l}^{+}$are infinitesimally equivalent to holomorphic discrete series representations.

Proof. Since the proof can be done as in the general case, we will only prove the representation theory assertion. As in the general case, we have that $\phi_{\nu,-l}(g)=\left\langle\pi_{\nu, l} 1_{l, \nu}, 1_{l, \nu}\right\rangle$, where $1_{l, \nu}($ kan $)=a^{-(\nu+\rho)} \tau_{l}(k)^{-1}$ belongs to $H^{\nu,+}$ (resp. $H^{\nu,-}$ ) if $l$ is even (resp. $l$ odd).

If we denote $k(\theta)=\left[\begin{array}{cc}e^{\mathbf{i} \theta} & 0 \\ 0 & e^{-\mathbf{i} \theta}\end{array}\right]$, then $\tau_{l}(k(\theta))=e^{-\mathbf{i} l \theta}$, and therefore $1_{\nu, l}(k(\theta)$ an $)=a^{-(\nu+\rho)} e^{\mathbf{i} l \theta}$. On the other hand, since $\mathfrak{g}$ is isomorphic to $\widetilde{\mathfrak{g}}=\operatorname{sl}(2, \mathbb{R})$, for each $\nu \in \mathbb{C}, 1_{\nu, l}$ can be identified with the function of $S L(2, \mathbb{R})$ defined by

$$
\phi_{-l}\left(\left[\begin{array}{cc}
e^{-\mathbf{i} t} & 0 \\
0 & e^{-\mathbf{i} t}
\end{array}\right]\left[\begin{array}{cc}
e^{2 \mathbf{i} t} & e^{2 \mathbf{i} t} x \\
0 & 1
\end{array}\right]\left[\begin{array}{cc}
\cos \theta & \sin \theta \\
-\sin \theta & \cos \theta
\end{array}\right]\right)=e^{(\nu+1) t} e^{-\mathbf{i} l \theta} .
$$

This function belongs to $H(\nu)=\left\{f: S l(2, \mathbb{R}) \mapsto \mathbb{C}: f(a n k)=a^{\nu+1} f(k)\right.$, $\left.f_{\mid K} \in L^{2}(K)\right\}$ (see [7, p. 116]) and the ( $\left.\mathfrak{g}, K\right)$-modules of $H^{\nu, \pm}$ generated by $1_{\nu, l}$ are isomorphic to the $(\widetilde{\mathfrak{g}}, K)$-modules of $H(\nu)$ generated by $\phi_{-l}$.

We note that the difference in the sign (with [7]) is due to the different choices in the Iwasawa decompositions.

We thus have that $V_{\nu_{k, l}^{-}, l} \simeq \sum_{j=1}^{|l|-1+2 k}\left\langle\phi_{-(|l|+2(k-j))}\right\rangle$, and therefore $V_{\nu_{k, l}^{-}, l}$ is finite dimensional. If $\nu_{k, l}^{+} \neq 0$, then we obtain the discrete series:

$$
V_{\nu_{k, l}^{+}, l} \simeq \begin{cases}\sum_{\substack{j \equiv l(2) \\ j \leq-l+2 k}}\left\langle\phi_{j}\right\rangle \quad l>0 \\ \sum_{\substack{j \equiv l(2) \\ j \geq-l-2 k}}\left\langle\phi_{j}\right\rangle \quad l<0 .\end{cases}
$$


Finally, we can see that if $\nu=0$ is a pole then $l$ is odd, and therefore we obtain the so called 'Mock discrete series' or limit of discrete series representations:

$$
V_{0, l} \simeq \begin{cases}\sum_{\substack{j \equiv l(2) \\ j \leq-1}}\left\langle\phi_{j}\right\rangle \quad l>0 \\ \sum_{\substack{j \equiv l(2) \\ j \geq 1}}\left\langle\phi_{j}\right\rangle \quad l<0\end{cases}
$$

thus concluding the proof.

\section{References}

[1] A. Deitmar, Invariant operators on higher K-types, J. Reine Angew. Math., 412 (1990), 97-107, MR 92b:22013, Zbl 0712.43006.

[2] M. Eguchi and S. Koizumi, The explicit formula for the Harish-Chandra C-function of $S U(n, 1)$ for arbitrary irreducible representation of $K$ which contain one-dimensional K-types, Proc. Japan Acad., Ser A, 70 (1994), 256-259, MR 95i:22019, Zbl 0838.43008.

[3] Harish-Chandra, Differential equations and semisimple Lie groups, manuscript, 1960, in 'Collected Papers', Vol. III, 57-120, Springer Verlag, New York, 1984, MR 85e:01061c.

[4] G.J. Heckman and E.M. Opdam, Root system and hypergeometric functions I, Compositio Mathematicae, 64 (1987), 329-352, MR 89b:58192a, Zbl 0656.17006.

[5] A.W. Knapp, Representation Theory of Semisimple Groups, Princeton Univ. Press, Princeton, New Jersey, 1986, MR 87j:22022, Zbl 0604.22001.

[6] T. Koornwinder, Jacobi functions and analysis on noncompact semisimple Lie groups, in 'Special Functions: Group Theorical Aspects and Applications', Reidel, Dordrecht, 1984, 1-85, MR 86m:33018, Zbl 0584.43010.

[7] S. Lang, $S L(2, \mathbb{R})$, Springer-Verlag, New York, 1985, Zbl 0583.22001.

[8] R.J. Miatello and N.R. Wallach, The resolvent of the Laplacian on negatively curved locally symmetric spaces of finite volume, J. Differential Geom., 36 (1992), 663-698, MR 93i:58160, Zbl 0766.53044.

[9] R. Miatello and C.E. Will, The residues of the resolvent on Damek-Ricci spaces, Proc. Amer. Math. Soc., 128 (2000), 1221-1229, MR 2001i:22011, Zbl 0941.22005.

[10] H. Schlichtkrull, One dimensional $K$-types in finite dimensional representations of semisimple Lie groups: A generalization of Helgason's theorem, Math. Scand., 54 (1984), 279-294, MR 85i:22014, Zbl 0545.22015.

[11] N. Shimeno, The Plancherel formula for spherical functions with a one-dimensional K-type on a simply connected simple Lie group of Hermitian type, J. Funct. Anal., 121 (1994), 330-388, MR 95b:22036, Zbl 0830.43018.

[12] _ Eigenspaces of invariant differential operators on a homogeneous line bundle on a Riemannian symmetric space, J. Fac. Sci. Univ. Tokyo, Sect. I.A, Math., 37 (1990), 201-234, MR 91j:22008, Zbl 0713.58053. 
[13] N. Wallach, Harmonic Analysis on Homogeneous Spaces, Pure and Appl. Math., 19, Marcel Dekker Inc., New York, 1973, MR 58 \#16978, Zbl 0265.22022.

Received June 8, 2000 and revised May 10, 2001. This research was supported by a fellowship from CONICET and research grants from CONICET and SeCyT UNC (Argentina).

Ciem, FAMAF

Universidad NACIONAL DE Córdoba

5000 Córdoba

Argentina

E-mail address: cwill@mate.uncor.edu 